\title{
Isolated Vomiting due to a Unilateral Dorsal Vagal Complex Lesion
}

\author{
Setsu Sawai Ryuji Sakakibara Kazuaki Kanai Naoki Kawaguchi Tomoyuki Uchiyama \\ Tatsuya Yamamoto Takashi Ito Zhi Liu Takamichi Hattori \\ Department of Neurology, Chiba University, Chiba, Japan
}

Dear Sir,

Recent experimental studies have indicated a 'vomiting pattern generator' (formerly called the 'vomiting center') situated in the dorsal portion of the lateral reticular formation of the medulla, which mostly corresponds to the dorsal vagal complex (DVC) [1]. There is also a che- mosensitive trigger zone in the floor of the fourth ventricle in the area postrema, which lies just adjacent to the DVC $[2,3]$. When accompanied by vertigo or raised intracranial pressure, brain diseases often cause nausea and vomiting. However, isolated central vomiting is rare [4-8]. We here report the case of a young woman who had a unilateral DVC lesion due to central nervous system (CNS) lupus, which caused isolated vomiting, without vertigo or raised intracranial pressure.
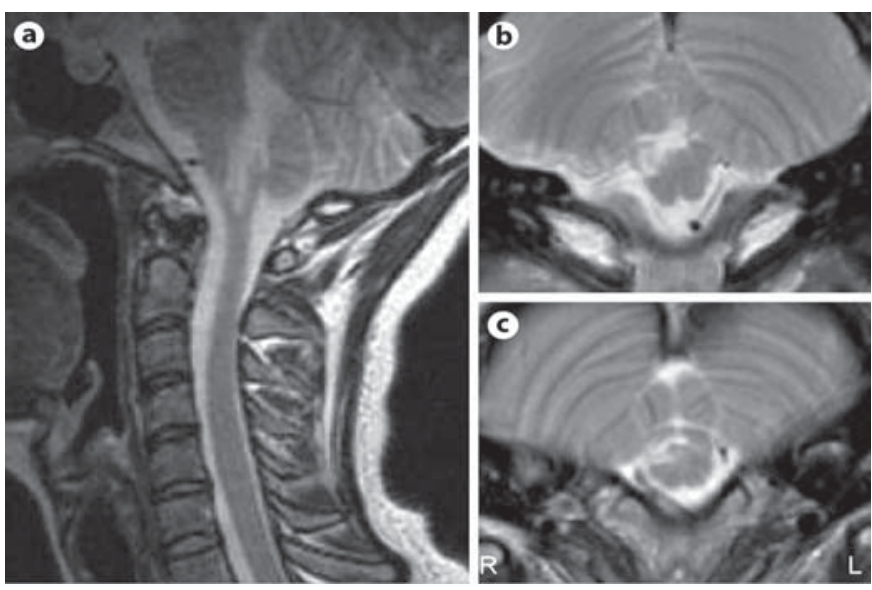

Fig. 1. Brain MRI of the patient. a $\mathrm{A} \mathrm{T}_{2}$-weighted sagittal image showed a high signal intensity lesion in the medulla. b, $\mathbf{c ~ T}_{2}$ weighted axial images showed a high signal intensity lesion in the right-side, dorsolateral medulla. Axial MR images are upside down in order to conform to the anatomical illustration below. d A figure illustrating the area of the lesion (hatched area) in the axial MRI and the location of the inferior vestibular nucleus and the dorsal vagal complex, corresponding to the vomiting pattern

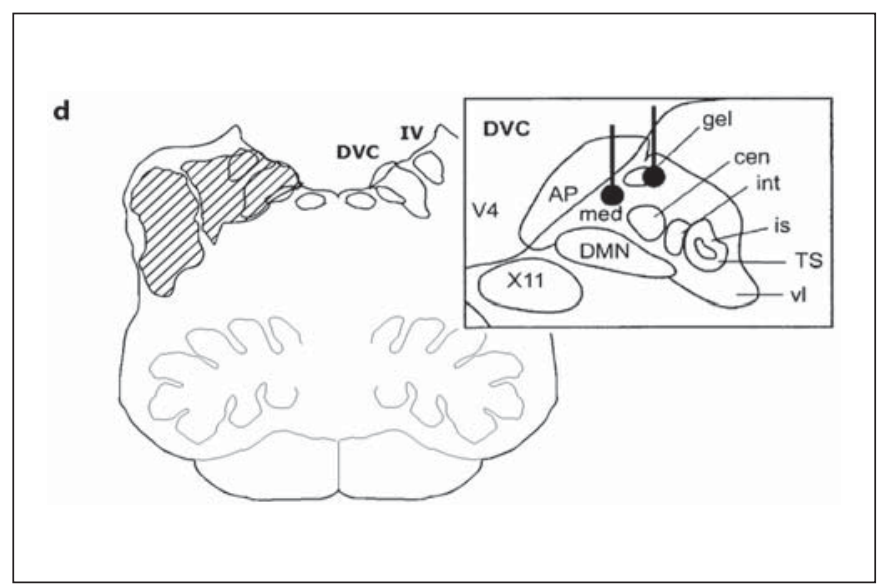

generator, which includes the area postrema, e.g. the chemosensitive trigger zone. DVC = Dorsal vagal complex; IV = inferior vestibular nucleus; AP = area postrema; med = medial NTS (nucleus tractus solitarius); gel = subnucleus gelatinosus; cen = subnucleus centralis; int = intermediate subnucleus; is = interstitial subnucleus; TS = tractus solitarius; $\mathrm{vl}=$ ventrolateral subnucleus of NTS; $\mathrm{DMN}=$ dorsal motor nucleus; $\mathrm{X} 11$ = hypoglossal nucleus; $\mathrm{V} 4$ = fourth ventricle. Modified from Hornby [1].

\section{KARGER}

Fax +41613061234 E-Mail karger@karger.ch www.karger.com
(C) 2006 S. Karger AG, Basel 0014-3022/06/0564-0246\$23.50/0

Accessible online at: www.karger.com/ene
Ryuji Sakakibara, MD

Neurology Department, Chiba University

1-8-1 Inohana Chuo-ku

Chiba 260-8670 (Japan)

Tel. +81 43226 2129, Fax +81 43226 2160, E-Mail sakakibara@faculty.chiba-u.jp 


\section{Case Report}

A 23-year-old woman had a transient episode of hallucination ('hearing a voice') and delusion ('a wiretap being attached in her house') - both lasting 1 week - as well as headache and $38^{\circ} \mathrm{C}$ fever - both lasting 2 days. Three months later, she came down with a $37^{\circ} \mathrm{C}$ fever and vomiting. The vomiting was not precipitated by eating and was associated with nausea. There was no morning vomiting. She had no vertigo or dizziness. With these symptoms, she was admitted to a local gastroenterological hospital. Normal results were found for a full blood count, biochemical profile, blood culture, chest X-ray, abdominal Xray, abdominal ultrasound scan, gastroscopy and computed tomography (CT) scans of the pelvis/abdomen, chest and cerebrum; the only abnormal findings were mild inflammatory markers and a positive antinuclear antibody titer of 320 . However, no definite diagnosis of collagen disease was made at that time, and the unexplained nausea and vomiting persisted, which remained refractory to antiemetics. She had no episodes of postural hypotension or urinary dysfunction. Two months after admission, she suddenly developed generalized convulsion and was then referred to us.

On admission to our hospital, she was slightly drowsy and disoriented. She had no apparent signs of raised intracranial pressure. Her cranial nerves were normal except for right pharyngeal paresis, although she had no dysphagia or dysarthria. There was a mild gaze-evoked nystagmus to the left, although she had no vertigo or dizziness sensation whatsoever. The neurological examination was otherwise normal. Three days after admission, she developed myalgia and arthralgia. Laboratory examination results showed increased C-reactive protein, but her white blood cell count was low (up to 2,100/ $\mathrm{mm}^{3}$ ). She had an increased anti-SSA antibody titer of 113.0, but all other autoantibodies were negative. No kidney dysfunction was seen. A cerebrospinal fluid examination and a brain magnetic resonance imaging (MRI) scan were performed just after the convulsion. These showed a mild increase in cell count of $32 / \mathrm{mm}^{3}$ (mononuclear:polymorphonuclear $=31: 1$ ) and total protein of $64 \mathrm{mg} / \mathrm{dl}$. The MRI scan, using $\mathrm{T}_{1^{-}}, \mathrm{T}_{2^{-}}$, diffusionweighted and fluid-attenuated inversion recovery sequences in axial planes, demonstrated an area of abnormal signal in- tensity in the right dorsolateral medulla that included the inferior vestibular nucleus and the DVC (fig. 1), in addition to multiple bilateral lesions in the cortical/ subcortical white matter (figure not shown). No brain edema was seen. Gadolinium contrast injection did not enhance the area, and the appearance was that of an inflammation. The diagnosis of systemic lupus erythematosus was made, based on arthralgia, leukocytopenia, increased antinuclear antibody and CNS involvement (CNS lupus) [9, 10]. She was started with methylprednisolone $1 \mathrm{~g} /$ day for 3 days (steroid pulse), resulting in prompt resolution of her drowsiness, nausea and vomiting. A follow-up brain MRI, obtained 1 month later, showed a decrease in the size of the dorsolateral medullary lesion as well as the complete disappearance of the cortical/white matter lesions. The latter lesions were thought to be reversible posterior leukoencephalopathy $[11,12]$.

\section{Discussion}

The most remarkable feature in our patient was the period of nausea and vomiting with neither vertigo, features of raised intracranial pressure nor organic causes in the gastrointestinal tract. Therefore, we can attribute her nausea and vomiting to the unilateral dorsolateral medullary lesion, which involved the DVC. Isolated central vomiting is rare, when it lacks vertigo or raised intracranial pressure. Such patients may see general physicians or gastroenterologists first, since nausea and vomiting commonly occur due to several causes: regurgitation, local infection, food poisoning, vertigo, motion sickness, neurotropic drugs (e.g. levodopa, antidepressants, opiates) or chemotherapy [3]. If such conditions have been excluded, CNS causes should be considered.

Only a few similar cases have been described in the literature. Baker and Bernat [4] described a patient with metastatic malignant melanoma in the dorsolateral pons by CT scan, who had gait ataxia and diplopia. Wood et al. [5] described a patient with a $2-\mathrm{cm}$ diameter astrocytoma in the medulla detected by MRI, who had headache, hiccup, decreased gut motility, postural hypotension and sudomotor failure. Mann et al. [6] described a patient with low-grade brain tumor in the midbrain detected by MRI. Grossmann et al. [7] described a patient with cavernoma in the medulla to the upper cervical cord detected by MRI. Torrealba et al. [8] reported 4 cases of mass lesions in the posterior fossa. Patients with ependymomas of the fourth ventricle also vomit periodically [13]. A close viewing of the MRI lesion in our case suggested that it involved the inferior vestibular nucleus, which might be related to her gaze-evoked nystagmus, although she did not have vertigo at all. The disparity between nystagmus and subjective dizziness is remarkable, and many patients with dorsal medullary lesions have nystagmus but are not dizzy. In addition, the lesion involved the DVC (fig. 1). The DVC is an area where sequential somatoautonomic vomiting behaviors are generated and where sensory inputs are integrated, including those from the vagal, vestibular and limbic complexes. The lesion also involves a chemosensitive trigger zone just adjacent to the DVC. The chemosensitive trigger zone lies outside the blood-brain barrier and senses various kinds of chemicals [14]. A unilateral DVC lesion should be listed in the differential diagnosis of isolated vomiting.

\section{References}

1 Hornby PJ: Central neurocircuitry associated with emesis. Am J Med 2001;111:106S$112 \mathrm{~S}$.

2 Andrews PLR, Horn CC: Signals for nausea and emesis: implications for models of upper gastrointestinal diseases. Auton Neurosci 2006;125:100-115.

3 Quigley EM, Hasler WL, Parkman HP: AGA technical review on nausea and vomiting. Gastroenterology 2001;120:263-286.

4 Baker PC, Bernat JL: The neuroanatomy of vomiting in man: association of projectile vomiting with a solitary metastasis in the lateral tegmentum of the pons and the middle cerebellar peduncle. J Neurol Neurosurg Psychiatry 1985;48:1165-1168.

5 Wood JR, Camilleri M, Low PA, Malagelada JR: Brainstem tumour presenting as an upper gut motility disorder. Gastroenterology 1985;89:1411-1414.

6 Mann SD, Danesh BJ, Kamm MA: Intractable vomiting due to a brainstem lesion in the absence of neurological signs or raised intracranial pressure. Gut 1998;42:875-877.

7 Grossmann D, Burtzlaff C, Griefahn B, Stenger RD, Wiersbitzky H, Wagner W, Lauffer $\mathrm{H}$ : Cavernoma of the medulla oblongata mimicking 'anorexia nervosa' - A case report (in German with English abstract). Klin Pädiatr 2002;214:41-44.

8 Torrealba G, Del Villar S, Arriagada P: Protracted vomiting as the presenting sign of posterior fossa mass lesions. J Neurol Neurosurg Psychiatry 1987;50:1539-1541. 
9 Uramoto KM, Michet CJ Jr, Thumboo J, Sunku J, O'Fallon WM, Gabriel SE: Trends in the incidence and mortality of systemic lupus erythematosus, 1950-1992. Arthritis Rheum 1999;42:2021-2022.

10 Jennekens FG, Kater L: The central nervous system in systemic lupus erythematosus. 1. Clinical syndromes: a literature investigation. Rheumatology 2002;41:605-618.
11 Shim KC, Choi HJ, Bae YD, Lee JC, Lee EB, Song YW: Reversible posterior leukoencephalopathy syndrome in systemic lupus erythematosus with thrombocytopenia treated with cyclosporine. J Clin Rheumatol 2005; 11:127-128.

12 Obeid T, Shami A, Karsou S: The role of seizures in reversible posterior leukoencephalopathy. Seizure 2004;13:277-281.
13 Akyuz C, Emir S, Akalan N, Soylemezoglu F, Kutluk T, Buyukpamukcu M: Intracranial ependymoma in childhood: a retrospective review of sixty-two children. Acta Oncol 2000;39:97-100.

14 Tonini M, Cipollina L, Poluzzi E, Crema F, Corazza GR, de Ponti F: Clinical implications of enteric and central $\mathrm{D}_{2}$ receptor blockade by antidopaminergic gastrointestinal prokinetics. Aliment Pharmacol Ther 2004;19:379-390. 